\title{
Global relationship between parent and child obesity: a systematic review and meta-analysis
}

\author{
Ju Suk Lee, MD, PhD ${ }^{1}$, Mi Hyeon Jin², Hae Jeong Lee, MD, PhD ${ }^{1}$ \\ ${ }^{1}$ Department of Pediatrics, Samsung Changwon Hospital, Sungkyunkwan University School of Medicine, Changwon, Korea; ${ }^{2}$ Department of Biostatistics, Samsung \\ Changwon Hospital, Sungkyunkwan University School of Medicine, Changwon, Korea
}

Background: The growing prevalence of overweight and/or obese children is an important public health problem in both developed and developing countries. Although the association of obesity between parents and their children is well known, its underlying mechanisms are not well established.

Purpose: This meta-analysis examined parent-child (PC) relationships in obesity and identified factors such as world region and country income level that may influence this relationship.

Methods: We identified all related studies published between January 1, 2015 and May 31, 2020 by conducting a literature search using the MeSH terms "obesity," "overweight," "body mass index," "parent," "child," "associate," and "relate" in the PubMed database in English.

Results: The meta-analysis of 23 studies that reported an odds ratio (OR) for parent and child obesity associations found a significant association between parents and children who were overweight or obese (pooled OR, 1.97; 95\% confidence interval, 1.85-2.10). A meta-regression analysis was used to examine the sources of interstudy heterogeneity. The association between parent and child obesity was higher in Asia than in Europe and the Middle East and higher in highincome countries than in middle-or low-income countries. In addition, a higher association between parent and child obesity was found when both parents were obese than when only the father or mother was obese. This study from multiple countries indicates a significant $\mathrm{PC}$ relationship in weight status that varies according to PC pair type, parent and child weight statuses, world region, and country income level.

Conclusion: These results demonstrate that the risk of childhood obesity is greatly influenced by parental weight status and indicate that parents could play an important role in preventing child obesity.

Key words: Obesity, Child, Parent

\begin{abstract}
Key message
Question: Are parent and child obesity correlated worldwide?

Finding: Overweight and obese status of parents and children were significantly associated worldwide. The association between parent and child obesity was stronger in Asia than in Europe and the Middle East, and in high-income than in middle- and low-income countries.

Meaning: Childhood obesity is highly influenced by parental weight status, indicating that parents could play an important role in its prevention.
\end{abstract}

\section{Introduction}

Childhood obesity $(\mathrm{OB})$ is a growing global health issue. Worldwide, the age-standardized prevalence of $\mathrm{OB}$ in adults (18 years and older) has increased 1.5 times since the year 2000, and the crude prevalence in children aged 5 to19 years more than doubled (from $2.9 \%$ to $6.8 \%$ ) by $2016 .{ }^{1)}$ The percentage of overweight (OW) children younger than 5 years was estimated to be around $5.6 \%$ or 38.3 million in 2019, compared to about $2.9 \%$ or 30.3 million in $\left.2000 .{ }^{1,2}\right)$ The prevalence of OW and obese conditions continues to increase in adults and children. In $2016,39 \%$ of adults aged 18 years and over $(39 \%$ of men and $40 \%$ of women) were OW, and about $13 \%$ ( $11 \%$ of men and $15 \%$ of women) were obese. ${ }^{3)}$

$\mathrm{OB}$ is a multifactorial chronic disease influenced by biological, behavioral, and environmental factors. ${ }^{4}$ Childhood-onset $\mathrm{OB}$ frequently leads to adult $\mathrm{OB}$ and is associated with increased risk of lifestyle-related diseases including diabetes, hypertension, dyslipidemia, and cardiovascular disease, which in turn are associated with a higher chance of premature mortality and disability. ${ }^{5)} \mathrm{OB}$ can affect a child's immediate health, educational attainment, mental health, and quality of life.

Many studies have described the association between a parent's OW or obese status and that of their child, but the underlying mechanisms are not well established. ${ }^{6}$

Corresponding author: Hae Jeong Lee, MD, PhD. Department of Pediatrics, Samsung Changwon Hospital, Sungkyunkwan University School of Medicine, 158, Paryong-ro, Masanhoewon-gu, Changwon 51353, Korea 


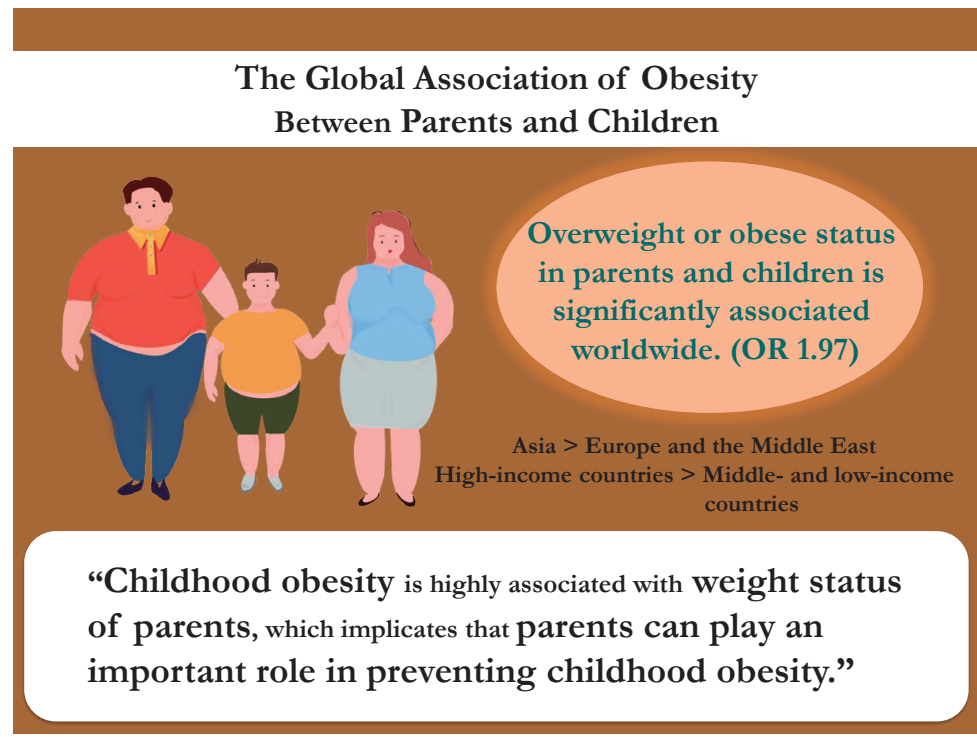

Graphic abstract

It is important to understand the causes and consequences of childhood OB. This paper studied parent-child (PC) OB relationships according to type of $\mathrm{PC}$ pair, parent and child weight status, and level of economy in the country. This paper serves as a systematic review that more specifically addresses childhood OB by presenting an overview of the global epidemiology, consequences, and etiopathogenesis relating to parental OB.

\section{Methods}

\section{Search strategy}

Using the MeSH terms obesity, overweight, body mass index (BMI), parent, child, associate, and relate, we searched the PubMed database in English from 1 January 2015 to 31 May 2020 as follows: ((obesity[Title]) OR (overweight[Title])) OR (body mass index[Title])) OR (BMI[Title])) AND (child*[Title])) AND (parent*[Title/Abstract])) AND ((associate*[Title/Abstract]) OR (relate*[Title/Abstract])) AND ("2015/01/01"[Date - Publication] : "2020/05/31"[Date - Publication]). The initial search included 837 articles. We reviewed titles and abstracts and excluded 752 articles that either did not describe parent and child OB relationships, had a total sample size $<200$, or were review papers. The full text of 85 articles was reviewed, and 23 papers were selected for the final analysis based on the inclusion criteria described below. Fig. 1 shows a flow diagram of the literature search.

\section{Inclusion criteria}

Articles were included if they met all of the following conditions: (1) both parents' and children's weight statuses were categorized based on BMI; (2) children were between 2-18 years old; (3) total sample size $\geq 200$; (4) results were described as the parent and child $\mathrm{OB}$ correlation (odds ratio, OR); and (5)

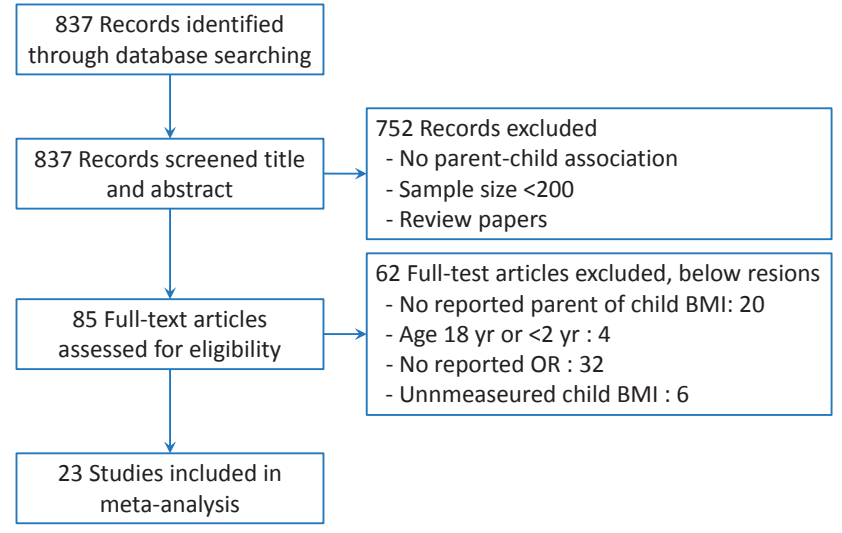

Fig. 1. Flow diagram of the literature search.

child weight and height were directly measured. The following data were included: authors and publication year, study design, study country, number of participants, age, BMI-based parent and child weight status, and key findings on the parent and child correlation in $\mathrm{OB}$.

\section{Exclusion criteria}

Articles were excluded if they met any of the following conditions: (1) the parents' or child's weight status was not based on BMI; (2) participants were $>18$ or $<2$ years old; (3) total sample size $<200$; (4) results for the OB relationship were not described using OR; and (5) parent- or self-reported child weight and height were used.

\section{Data extraction}

Data were extracted and summarized into a standardized data extraction form base on PRISMA (Preferred Reporting Items for Systematic reviews and Meta-Analyses) guidelines.7) Data extracted included first author, publication year, study design, study title, study country, world bank ranking, sample size, PC pair, parent and child weight status, and main results. In total, 
102 data points of PC associations in OW and/or OB were collected from the 23 studies. All studies reported ORs, and the meta-analysis was performed on the reported ORs. We included ORs for mutually exclusive specific categories of a PC pair (e.g., father-boy [FB], mother-girl [MG]) as well as general PC pairs.

\section{Definitions of OW and obese}

The weight statuses of participants were classified as obese, OW, or normal weight, for which BMI cutoffs were used to define weight status across children and adults and throughout articles from different countries. OB is most often defined by the BMI, a mathematical formula of weight-to-height index. BMI $\left(\mathrm{kg} / \mathrm{m}^{2}\right)$ is calculated as weight $(\mathrm{kg})$ divided by height squared $\left(\mathrm{m}^{2}\right)$. In pediatric age groups, $\mathrm{OB}$ is diagnosed when $\mathrm{BMI}$ is higher than the 95 th percentile per age and sex, and BMI over the 85th but less than the 95th percentile for age and sex is considered OW. ${ }^{8)}$ Because a child's body composition varies with age and sex, a child's weight status is determined using age- and sex-specific percentiles for BMI, different from those used for adults. ${ }^{9)}$ The International Obesity Task Force (IOTF) has developed an international standard growth chart that allows prevalence to be compared globally. ${ }^{10)}$ However, many countries use their own country-specific growth charts. Most of the articles classified weight status according to the World Health Organization (WHO) BMI cutoff criteria, with the weight statuses of adults classified as follows: normal weight $\left(\mathrm{BMI}<25 \mathrm{~kg} / \mathrm{m}^{2}\right)$, OW (BMI 25-29.9 kg/m²), and obese (BMI $\left.\geq 30 \mathrm{~kg} / \mathrm{m}^{2}\right){ }^{11}$

\section{Types of $P C$ pairs and weight status combinations}

A total of 9 PC pairs were classified into 3 categories: (1) PC included PC, parent-boy, and parent-girl; (2) father-child (FC) included FC, FB, and father-girl; (3) mother-child (MC) included MC, mother-boy, and MG.

Ten types of PC weight status combinations were used as following: OW-OW, OB-OB, OW and OB (OWOB)-OWOB, OWOB-OB, OWOB-OW, OB-OW, OW-OB, BMI-OB, BMI$\mathrm{OW}$, and BMI-OWOB.

The most commonly reported combinations of PC weight status (parental weight status-child's weight status) were OWOW, OB-OB, and OWOB-OB, representing about $54.9 \%$ of the total data points. Of the 23 studies with parents, 6 used information from one parent only.

\section{Quality assessment and risk of bias}

The risk of bias for each included study was assessed using the RoBANS (Risk of Bias Assessment Tool for Non-randomized Studies). ${ }^{12}$ Selection, performance, detection, attrition, and reporting biases were assessed and classified as low, high, and unclear risk of bias. The results of the risk of bias assessment are shown in Fig. 2. There was a high risk of performance biases because the data were obtained through self-reported questionnaire.

\section{Statistical analysis}

In all studies, 9 PC pairs and $10 \mathrm{PC}$ weight status combinations were used.

We performed a meta-analysis according to the reported 102 ORs from 23 studies. Studies combined in a meta-analysis have differences in design, so we used a random-effects model to account for differences in the underlying study effects, which include a heterogeneity variance parameter. We fitted metaregression models with random effects and calculated pooled estimates with confidence intervals of effect size for the $\mathrm{PC} \mathrm{OB}$ correlation. There was significant heterogeneity for the effect size in the $\mathrm{PC} \mathrm{OB}$ relation (Cochrane $\mathrm{Q}=2295.87$, degrees of freedom $\left.[d f]=101, P<0.001 ; I^{2}=95.6 \%\right)$. The meta-regression analysis was used to determine which factors explained the heterogeneity. We considered child age, type of PC pair, parent and child weight status, world region, and country income level as covariates. Other than the child's age, all other factors were important predictors of the PC association for OB. The differences in pooled OR estimates in the PC OB relationship by PC pair are shown in separate forest plots. All analyses were performed using Stata Release 14.0. Statistical significance was set at $P<0.05$.

\section{Country income level}

The countries included in the 23 studies were classified into 3 categories according to level of national income, measured by gross national income per capita (GNI) using the World Bank Atlas method. For the current 2021 fiscal year, low-income economies are defined as those with a GNI per capita of $\$ 1,035$ or less in 2019; lower middle-income economies are those with a GNI per capita between $\$ 1,036$ and $\$ 4,045$; upper middleincome economies are those with a GNI per capita between $\$ 4,046$ and $\$ 12,535$; and high-income economies are those with a GNI per capita of $\$ 12,536$ or more. ${ }^{13)}$ Of the 24 countries included in the 23 studies, Korea, Spain, USA, Hong Kong (China), Germany, Italy, Australia, Canada, Finland, Portugal, UK, and Japan were classified as high-income countries; China (except Hong Kong), Brazil, Thailand, Turkey, Palestine, Argentina, Iran, Cameroon, Indonesia, Colombia, India, and South Africa were classified as middle-income countries; and Kenya was classified as a low-income country.

\section{Results}

\section{General characteristics}

The characteristics and findings of the 23 studies are shown in Table $1 .{ }^{14-36)}$ Most studies were cross-sectional $(\mathrm{n}=21)$, with sample sizes of the PC pairs varying from 486 to $42,431 .{ }^{15,32)}$ Most of the studies included school-aged children $(n=20)$, though 3 focused on preschool children under the age of 5 . For income status, 9 studies were conducted in high-income countries, 13 studies were conducted in middle-income countries, and one study included 12 countries from low-income to 
Table 1. General characteristics of included studies

\begin{tabular}{|c|c|c|c|c|c|c|c|c|c|}
\hline Study & $\begin{array}{l}\text { Study } \\
\text { design }\end{array}$ & Title & $\begin{array}{l}\text { World } \\
\text { region/ } \\
\text { country }\end{array}$ & $\begin{array}{l}\text { World } \\
\text { bank } \\
\text { ranking }\end{array}$ & $\begin{array}{l}\text { Sample } \\
\text { size }\end{array}$ & $\begin{array}{l}\text { Child } \\
\text { age } \\
\text { (yr) }\end{array}$ & $\begin{array}{l}\text { Weight } \\
\text { related } \\
\text { outcomes } \\
\text { of parent }^{\text {a) }}\end{array}$ & $\begin{array}{l}\text { Weight } \\
\text { related } \\
\text { outcomes } \\
\text { of children }{ }^{\text {b) }}\end{array}$ & $\begin{array}{l}\text { Main results PC relationship } \\
\text { in obesity: OR }(95 \% \mathrm{CI})\end{array}$ \\
\hline $\begin{array}{l}\text { Cheng et al., }{ }^{14)} \\
2020\end{array}$ & $\begin{array}{l}\text { Cross- } \\
\text { sectional }\end{array}$ & $\begin{array}{l}\text { The associations of specific } \\
\text { school-and individual-level } \\
\text { characteristics with obesity } \\
\text { among primary school } \\
\text { children }\end{array}$ & $\begin{array}{l}\text { Beijing, } \\
\text { China }\end{array}$ & $\begin{array}{l}\text { Upper } \\
\text { middle } \\
\text { income }\end{array}$ & 2,201 & $10 \pm 0.41$ & OWOB & $\begin{array}{l}\text { OB } \\
\text { IOTF }\end{array}$ & $\begin{array}{l}F(O W O B) C: 2.09(1.63-2.69) \\
\text { M (OWOB) C: } 2.77(2.12-3.61)\end{array}$ \\
\hline $\begin{array}{l}\text { Caixeta and } \\
\text { Amato }^{15)} \\
2020\end{array}$ & $\begin{array}{l}\text { Cross- } \\
\text { sectional }\end{array}$ & $\begin{array}{l}\text { Factors associated with } \\
\text { overweight and abdominal } \\
\text { obesity in Brazilian school- } \\
\text { aged children: a compre- } \\
\text { hensive approach }\end{array}$ & Brazil & $\begin{array}{l}\text { Upper } \\
\text { middle } \\
\text { income }\end{array}$ & 486 & $6-8$ & $\mathrm{BMI}$ & $\begin{array}{l}\text { OWOB } \\
\text { CDC } \\
\text { reference }\end{array}$ & MC: 1.05 (1.01-1.10) \\
\hline $\begin{array}{l}\text { Lee et. al., }{ }^{16)} \\
2020\end{array}$ & $\begin{array}{l}\text { Cross- } \\
\text { sectional }\end{array}$ & $\begin{array}{l}\text { Variability in sociodemogra- } \\
\text { phic factors and obesity in } \\
\text { Korean children: a cross- } \\
\text { sectional analysis of Korea } \\
\text { National Health and Nutri- } \\
\text { tion Examination survey } \\
\text { data (2007-2015) }\end{array}$ & Korea & $\begin{array}{l}\text { High } \\
\text { income }\end{array}$ & 14,482 & $2-18$ & OW, OB & $\begin{array}{l}\text { OB } \\
\text { Korean } \\
\text { growth } \\
\text { chart }\end{array}$ & $\begin{array}{l}\text { M (OW) C (2-6 yr): } 2.25(1.34-3.78) \\
\text { M (OB) C (2-6 yr): } 4.67(2.35-9.26) \\
F(O W) C(2-6 y r): 2.59(1.70-3.97) \\
F(O B) C(2-6 y r): 6.07(2.86-12.91) \\
\text { M (OW) C (7-12 yr): } 2.47(1.73-3.52) \\
\text { M (OB) C (7-12 yr): } 3.47(1.89-6.38) \\
F(O W) C(7-12 y r): 2.47(1.76-3.47) \\
F(O B) C \text { (7-12 yr): } 2.25(1.02-4.98) \\
\text { M (OW) C (13-18 yr): } 2.32(1.52-3.52) \\
\text { M (OB) C (13-18 yr): } 3.82(2.09-6.98) \\
F(O W) C(13-18 y r): 2.07(1.45-2.98) \\
F(O B) C(13-18 y r): 5.30(2.78-10.12)\end{array}$ \\
\hline $\begin{array}{l}\text { Nonboonyawat } \\
\text { et al., }{ }^{17)} 2019\end{array}$ & $\begin{array}{l}\text { Cross- } \\
\text { sectional }\end{array}$ & $\begin{array}{l}\text { Prevalence and associates } \\
\text { of obesity and overweight } \\
\text { among school-age children } \\
\text { in rural community }\end{array}$ & Thailand & $\begin{array}{l}\text { Upper } \\
\text { middle } \\
\text { income }\end{array}$ & 1,749 & $6-18$ & $\mathrm{BMI}$ & $\begin{array}{l}\text { OWOB } \\
\text { WHO } \\
\text { reference }\end{array}$ & $\begin{array}{l}\text { MC: } 1.07(1.02-1.12) \\
\text { FC: } 0.98(0.93-1.03)\end{array}$ \\
\hline $\begin{array}{l}\text { Yardim et al., } \\
2019\end{array}$ & $\begin{array}{l}\text { Cross- } \\
\text { sectional }\end{array}$ & $\begin{array}{l}\text { Prevalence of childhood obe- } \\
\text { sity and related parental } \\
\text { factors }\end{array}$ & $\begin{array}{l}\text { Ankara, } \\
\text { Turkey }\end{array}$ & $\begin{array}{l}\text { Upper } \\
\text { middle } \\
\text { income }\end{array}$ & 2,066 & $9-11$ & OWOB & $\begin{array}{l}\text { OW, OB } \\
\text { WHO } \\
\text { reference }\end{array}$ & $\begin{array}{l}F C(O W): 1.44(0.97-2.15) \\
\text { FC (OB): } 1.94(1.18-3.18) \\
\text { MC (OW): } 1.36(0.81-2.30) \\
\text { MC (OB): } 2.07(1.13-3.79) \\
\text { PC (OW): } 2.05(1.39-3.02) \\
\text { PC (OB): } 3.80(2.3-6.28)\end{array}$ \\
\hline $\begin{array}{l}\text { Al-Lahham et } \\
\text { al., }{ }^{19)} 2019\end{array}$ & $\begin{array}{l}\text { Cross- } \\
\text { sectional }\end{array}$ & $\begin{array}{l}\text { Prevalence of underweight, } \\
\text { overweight and obesity } \\
\text { among school-age children } \\
\text { and the associated risk fac- } \\
\text { tors: a cross sectional study }\end{array}$ & Palestine & $\begin{array}{l}\text { Lower } \\
\text { middle } \\
\text { income }\end{array}$ & 1,320 & $6-12$ & $\mathrm{BMI}$ & $\begin{array}{l}\text { OW, OB } \\
\text { CDC } \\
\text { reference }\end{array}$ & $\begin{array}{l}F C(O W): 1.0(0.92-1.08) \\
F C(O B): 1.09(0.98-1.22)\end{array}$ \\
\hline $\begin{array}{l}\text { Orden et al., } \\
2019\end{array}$ & $\begin{array}{l}\text { Cross- } \\
\text { sectional }\end{array}$ & $\begin{array}{l}\text { Short sleep and low milk } \\
\text { intake are associated with } \\
\text { obesity in a community of } \\
\text { school aged children }\end{array}$ & Argentina & $\begin{array}{l}\text { Upper } \\
\text { middle } \\
\text { income }\end{array}$ & 1,366 & $6-12$ & $\begin{array}{l}\text { BMI } \\
\text { IOTF }\end{array}$ & $\begin{array}{l}\text { OWOB, OW, } \\
\text { OB } \\
\text { IOTF }\end{array}$ & $\begin{array}{l}\text { FC (OWOB): } 1.52(1.24-1.68) \\
\text { MC (OWOB): } 1.65(1.37-1.98) \\
\text { FC (OW): } 1.34(1.04-1.68) \\
\text { MC (OW): } 1.30(1.06-1.60) \\
\text { FC (OB): } 1.48(1.11-1.99) \\
\text { MC (OB): } 1.83(1.43-2.34)\end{array}$ \\
\hline $\begin{array}{l}\text { Ejtahed et } \\
\text { al., }^{21)} 2018\end{array}$ & $\begin{array}{l}\text { Cross- }^{-} \\
\text {sectional }\end{array}$ & $\begin{array}{l}\text { Association of parental obe- } \\
\text { sity with cardiometabolic } \\
\text { risk factors in their children: } \\
\text { The CASPIAN-V study }\end{array}$ & Iran & $\begin{array}{l}\text { Upper } \\
\text { middle } \\
\text { income }\end{array}$ & 14,400 & $7-18$ & OW, OB & $\begin{array}{l}\text { OW, OB } \\
\text { Iran } \\
\text { reference }\end{array}$ & $\begin{array}{l}P(O W) C(O W): 1.30(1.17-1.44) \\
P(O B) C(O W): 1.46(1.29-1.65) \\
P(O W) C(O B): 1.36(1.18-1.59) \\
P(O B) C(O B): 1.60(1.35-1.90)\end{array}$ \\
\hline $\begin{array}{l}\text { Choukem et } \\
\text { al., }^{22)} 2017\end{array}$ & $\begin{array}{l}\text { Cross- } \\
\text { sectional }\end{array}$ & $\begin{array}{l}\text { Overweight and obesity in } \\
\text { children aged } 3-13 \text { years } \\
\text { in urban Cameroon: a } \\
\text { cross-sectional study of } \\
\text { prevalence and association } \\
\text { with socioeconomic status }\end{array}$ & Cameroon & $\begin{array}{l}\text { Lower } \\
\text { middle } \\
\text { income }\end{array}$ & 1,343 & $3-13$ & $\mathrm{BMI}$ & $\begin{array}{l}\text { overweight/ } \\
\text { obesity } \\
\text { (OWOB) } \\
\text { WHO } \\
\text { reference }\end{array}$ & MC: 1.06 (1.02-1.09) \\
\hline $\begin{array}{l}\text { Riaño-Galán et } \\
\text { al., }^{23)} 2017\end{array}$ & $\begin{array}{l}\text { Population- } \\
\text { based birth } \\
\text { cohort } \\
\text { study }\end{array}$ & $\begin{array}{l}\text { Proatherogenic lipid profile in } \\
\text { early childhood: association } \\
\text { with weight status at } 4 \\
\text { years and parental obesity }\end{array}$ & Spain & $\begin{array}{l}\text { High } \\
\text { income }\end{array}$ & 582 & 4 & OWOB & $\begin{array}{l}\text { OWOB } \\
\text { IOFT }\end{array}$ & $\begin{array}{l}\text { FC: } 3.73(1.90-7.34) \\
\text { MC: } 4.17(1.76-9.88) \\
\text { PC: } 5.10(2.5-10.40)\end{array}$ \\
\hline $\begin{array}{l}\text { Zhao et al., }{ }^{24)} \\
2017\end{array}$ & $\begin{array}{l}\text { Cross- } \\
\text { sectional }\end{array}$ & $\begin{array}{l}\text { Fast food consumption and } \\
\text { its associations with obesity } \\
\text { and hypertension among } \\
\text { children }\end{array}$ & $\begin{array}{l}\text { Beijing, } \\
\text { Shanghai, } \\
\text { Nanjing, } \\
\text { and Xian, } \\
\text { China }\end{array}$ & $\begin{array}{l}\text { Upper } \\
\text { middle } \\
\text { income }\end{array}$ & 1,626 & $7-16$ & $\mathrm{BMI}$ & $\begin{array}{l}\text { OB } \\
\text { Chinese } \\
\text { school- } \\
\text { age BMl } \\
\text { reference }\end{array}$ & MC: $1.11(1.06-1.17)$ \\
\hline
\end{tabular}


Table 1. Continued

\begin{tabular}{|c|c|c|c|c|c|c|c|c|c|}
\hline Study & $\begin{array}{l}\text { Study } \\
\text { design }\end{array}$ & Title & $\begin{array}{l}\text { World } \\
\text { region/ } \\
\text { country }\end{array}$ & $\begin{array}{l}\text { World } \\
\text { bank } \\
\text { ranking }\end{array}$ & $\begin{array}{l}\text { Sample } \\
\text { size }\end{array}$ & $\begin{array}{l}\text { Child } \\
\text { age } \\
\text { (yr) }\end{array}$ & $\begin{array}{l}\text { Weight } \\
\text { related } \\
\text { outcomes } \\
\text { of parent }^{\mathrm{a})}\end{array}$ & $\begin{array}{l}\text { Weight related } \\
\text { outcomes } \\
\text { of children }\end{array}$ & $\begin{array}{c}\text { Main results PC relationship } \\
\text { in obesity: OR }(95 \% \mathrm{Cl})\end{array}$ \\
\hline $\begin{array}{l}\text { Salahuddin et } \\
\text { al., }{ }^{25)} 2017\end{array}$ & $\begin{array}{l}\text { Cross- } \\
\text { sectional }\end{array}$ & $\begin{array}{l}\text { Predictors of severe obesity in } \\
\text { low-income, predominantly } \\
\text { Hispanic/Latino children: } \\
\text { the Texas childhood obesity } \\
\text { research demonstration } \\
\text { study }\end{array}$ & $\begin{array}{l}\text { Texas, } \\
\text { USA }\end{array}$ & $\begin{array}{l}\text { High } \\
\text { income }\end{array}$ & 517 & $2-12$ & $\begin{array}{c}\text { severe } \\
\text { obesity } \\
(\mathrm{BMl}>35 \\
\left.\mathrm{kg} / \mathrm{m}^{2}\right)\end{array}$ & $\begin{array}{l}\text { severe obesity } \\
\text { (BMI } \geq 120 \% \text { of } \\
\text { 95th percentile) } \\
\text { CDC reference }\end{array}$ & $\begin{array}{l}\text { MC (2-5 yr): } 2.67(1.10-6.47) \\
\text { MC (6-8 yr): } 1.58(0.74-3.35) \\
\text { MC (7-12 yr): } 4.12(1.84-9.23)\end{array}$ \\
\hline $\begin{array}{l}\text { Wang et al., }{ }^{26)} \\
2017\end{array}$ & $\begin{array}{l}\text { Cross- } \\
\text { sectional }\end{array}$ & $\begin{array}{l}\text { Prevalence of overweight in } \\
\text { Hong Kong Chinese children: } \\
\text { Its associations with family, } \\
\text { early-life development and } \\
\text { behaviors-related factors }\end{array}$ & $\begin{array}{l}\text { Hong } \\
\text { Kong, } \\
\text { China }\end{array}$ & $\begin{array}{l}\text { High } \\
\text { income }\end{array}$ & 894 & $9-12$ & $\begin{array}{l}\text { OWOB } \\
(\mathrm{BMI} \geq 25 \\
\left.\mathrm{kg} / \mathrm{m}^{2}\right)\end{array}$ & $\begin{array}{l}\text { OW } \\
\text { IOTF }\end{array}$ & $\begin{array}{l}\text { FC: } 1.72(1.18-2.52) \\
M C: 3.19(1.94-5.22)\end{array}$ \\
\hline $\begin{array}{l}\text { Bahreynian et } \\
\text { al., }{ }^{27)} 2017\end{array}$ & $\begin{array}{l}\text { Cross- } \\
\text { sectional }\end{array}$ & $\begin{array}{l}\text { Association between obesity } \\
\text { and parental weight status } \\
\text { in children and adolescents }\end{array}$ & Iran & $\begin{array}{l}\text { Upper } \\
\text { middle } \\
\text { income }\end{array}$ & 23,043 & $6-18$ & OW, OB & $\begin{array}{l}\mathrm{OB} \\
\text { WHO } \\
\text { reference }\end{array}$ & $\begin{array}{l}P(O W) B: 1.67(1.48-1.88) \\
P(O B) B: 2.55(2.23-2.92) \\
P(O W) G: 2.02(1.79-2.27) \\
P(O B) G: 3.46(3.04-3.94)\end{array}$ \\
\hline $\begin{array}{l}\text { Schüle et al., } \\
2016\end{array}$ & $\begin{array}{l}\text { Cross- }^{-} \\
\text {sectional }\end{array}$ & $\begin{array}{l}\text { Neighborhood socioeconomic } \\
\text { context, individual socioeco- } \\
\text { nomic position, and over- } \\
\text { weight in young children: a } \\
\text { multilevel study in a large } \\
\text { German city }\end{array}$ & Germany & $\begin{array}{l}\text { High } \\
\text { income }\end{array}$ & 3,499 & $5-7$ & OW, OB & $\begin{array}{l}\text { OW } \\
\text { IOTF }\end{array}$ & $\begin{array}{l}\text { M (OW) C: } 2.44(1.93-3.08) \\
\text { M (OB) C: } 3.08(2.19-4.34)\end{array}$ \\
\hline $\begin{array}{l}\text { Parrino et al., }{ }^{29)} \\
2016\end{array}$ & $\begin{array}{l}\text { Cross- } \\
\text { sectional }\end{array}$ & $\begin{array}{l}\text { Influence of early-life and pa- } \\
\text { rental factors on childhood } \\
\text { overweight and obesity }\end{array}$ & $\begin{array}{l}\text { Sicily, } \\
\text { Italy }\end{array}$ & $\begin{array}{l}\text { High } \\
\text { income }\end{array}$ & 1,521 & $9-14$ & OWOB & $\begin{array}{l}\text { OWOB } \\
\text { IOTF }\end{array}$ & $\begin{array}{l}\text { MC: } 2.33(1.80-3.01) \\
\text { FC: } 1.68(1.30-2.17)\end{array}$ \\
\hline $\begin{array}{l}\text { Rachmi et al., } \\
2016\end{array}$ & $\begin{array}{l}\text { Cross- } \\
\text { sectional }\end{array}$ & $\begin{array}{l}\text { Stunting, underweight and } \\
\text { overweight in children aged } \\
2.0-4.9 \text { years in Indonesia: } \\
\text { Prevalence trends and asso- } \\
\text { ciated risk factors }\end{array}$ & Indonesia & $\begin{array}{l}\text { Upper } \\
\text { middle } \\
\text { income }\end{array}$ & 4,101 & $2-4.9$ & OWOB & $\begin{array}{l}\text { OWOB } \\
\text { WHO } \\
\text { reference }\end{array}$ & $\begin{array}{l}\text { MC: } 1.88(1.24-2.87) \\
F C: 1.49(0.09-2.38)\end{array}$ \\
\hline $\begin{array}{l}\text { Muthuri et al., }{ }^{31)} \\
2016\end{array}$ & $\begin{array}{l}\text { Cross- } \\
\text { sectional }\end{array}$ & $\begin{array}{l}\text { Relationships between pa- } \\
\text { rental education and over- } \\
\text { weight with childhood over- }\end{array}$ & $\begin{array}{l}12- \\
\text { country } \\
\text { study }\end{array}$ & & 4,752 & $9-11$ & OW & $\begin{array}{l}\text { OW } \\
\text { WHO } \\
\text { reference }\end{array}$ & \\
\hline
\end{tabular}
weight and physical activity in 9-11-year-old children: results from a 12-country study

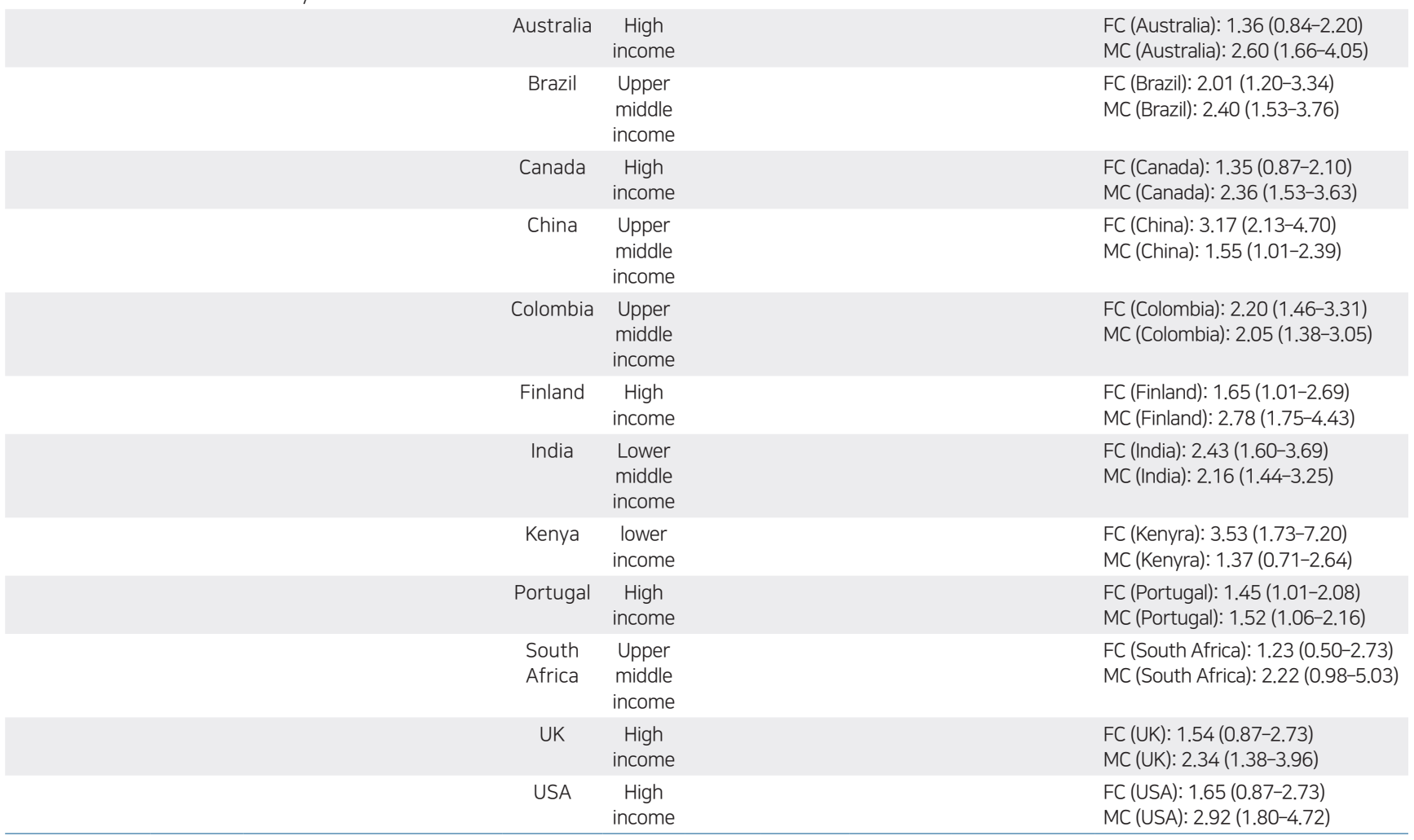




\begin{tabular}{|c|c|c|c|c|c|c|c|c|c|}
\hline Study & $\begin{array}{l}\text { Study } \\
\text { design }\end{array}$ & Title & $\begin{array}{l}\text { World } \\
\text { region/ } \\
\text { country }\end{array}$ & $\begin{array}{l}\text { World } \\
\text { bank } \\
\text { ranking }\end{array}$ & $\begin{array}{l}\text { Sample } \\
\text { size }\end{array}$ & $\begin{array}{l}\text { Child } \\
\text { age } \\
(y r)\end{array}$ & $\begin{array}{l}\text { Weight } \\
\text { related } \\
\text { outcomes } \\
\text { of parent }^{\text {a) }}\end{array}$ & $\begin{array}{l}\text { Weight related } \\
\text { outcomes } \\
\text { of children }\end{array}$ & $\begin{array}{l}\text { Main results PC relationship } \\
\text { in obesity: OR }(95 \% \mathrm{CI})\end{array}$ \\
\hline $\begin{array}{l}\text { Lombardo et } \\
\text { al., }^{32)} 2015\end{array}$ & $\begin{array}{l}\text { Cross- } \\
\text { sectional }\end{array}$ & $\begin{array}{l}\text { Severe obesity prevalence in 8- } \\
\text { to 9-year-old Italian children: a } \\
\text { large population-based study }\end{array}$ & Italy & $\begin{array}{l}\text { High } \\
\text { income }\end{array}$ & 42,431 & $8-9$ & OW, OB & $\begin{array}{l}\text { Severe obese } \\
\text { WHO } \\
\text { reference } \\
\text { (BMl>3 SD) }\end{array}$ & $\begin{array}{l}\text { P (OW) C: } 5.28 \text { (4.17-6.70) } \\
\text { P (OB) C: } 16.25 \text { (11.58-22.82) } \\
\text { P (OW) B: } 5.13(3.90-6.73) \\
\text { P (OB) B: } 13.13(8.67-19.89) \\
P(O W) \text { G: } 5.77(3.44-9.68) \\
P(O B) G: 24.3(13.07-45.15)\end{array}$ \\
\hline \multirow[t]{2}{*}{$\begin{array}{l}\text { Parikka et } \\
\text { al., }^{33)} 2015\end{array}$} & $\begin{array}{l}\text { Cross- } \\
\text { sectional }\end{array}$ & $\begin{array}{l}\text { Associations between parental } \\
\text { BMI, socioeconomic factors, } \\
\text { family structure and overweight } \\
\text { in Finnish children: a path model } \\
\text { approach }\end{array}$ & Finland & $\begin{array}{l}\text { High } \\
\text { income }\end{array}$ & 2,573 & $3-8$ & BMI & $\begin{array}{l}\text { OW } \\
\text { IOTF }\end{array}$ & $\begin{array}{l}\text { MB (3-8 yr): } 1.12(1.08-1.16) \\
\text { MG (3-8 yr): } 1.09(1.06-1.12) \\
\text { FB (3-8 yr): } 1.14(1.10-1.2) \\
\text { FG (3-8 yr): } 1.11(1.06-1.15)\end{array}$ \\
\hline & & & & & 1,836 & $11-16$ & & & $\begin{array}{l}\text { MB (11-16 yr): } 1.10(1.06-1.15) \\
\text { MG (11-16 yr): } 1.10(1.06-1.14) \\
\text { FB (11-16 yr): } 1.13(1.08-1.19) \\
\text { FG (11-16 yr): } 1.08 \text { (1.03-1.13) }\end{array}$ \\
\hline $\begin{array}{l}\text { Kachi et al., } \\
2015\end{array}$ & $\begin{array}{l}\text { Cross- } \\
\text { sectional }\end{array}$ & $\begin{array}{l}\text { Socioeconomic status and over- } \\
\text { weight: A population-based } \\
\text { cross-sectional study of Japanese } \\
\text { children and adolescents }\end{array}$ & Japan & $\begin{array}{l}\text { High } \\
\text { income }\end{array}$ & $\begin{array}{l}397 \\
397\end{array}$ & $\begin{array}{r}6-11 \\
12-18\end{array}$ & $\begin{array}{l}\text { OWOB } \\
(\mathrm{BMI} \geq 25 \\
\left.\mathrm{kg} / \mathrm{m}^{2}\right)\end{array}$ & $\begin{array}{l}\text { OW } \\
\text { IOTF }\end{array}$ & $\begin{array}{l}\text { MC (6-11 yr): } 2.72(1.30-5.71) \\
\text { MC (12-18 yr): } 3.47(1.49-8.07)\end{array}$ \\
\hline $\begin{array}{l}\text { Zong et al., } \\
2015\end{array}$ & $\begin{array}{l}\text { Population- } \\
\text { based, 1:1 } \\
\text { matched }\end{array}$ & $\begin{array}{l}\text { Family-related risk factors of } \\
\text { obesity among preschool children }\end{array}$ & China & $\begin{array}{l}\text { Upper } \\
\text { middle } \\
\text { income }\end{array}$ & 1,844 & $3-4$ & $\begin{array}{l}\text { OWOB } \\
(\mathrm{BMl} \geq 24 \\
\left.\mathrm{kg} / \mathrm{m}^{2}\right)\end{array}$ & $\begin{array}{l}\text { OB } \\
\text { IOTF }\end{array}$ & $\begin{array}{l}\text { FC (1996): } 2.33(1.28-4.23) \\
\text { MC (1996): } 2.49(1.31-4.76)\end{array}$ \\
\hline
\end{tabular}

\begin{tabular}{|c|c|c|c|c|c|c|c|c|}
\hline & & & & 3,298 & & & & $\begin{array}{l}\text { FC (2006): } 2.12(1.63-2.76) \\
\text { MC (2006): } 1.52(1.09-2.15)\end{array}$ \\
\hline $\begin{array}{l}\text { Wan et al., } \\
2015\end{array}$ & $\begin{array}{l}\text { Cross- } \\
\text { sectional }\end{array}$ & $\begin{array}{l}\text { Is parental body weight related Shanghai, } \\
\text { with their children's overweight China } \\
\text { and obesity in Gao Hang Town, } \\
\text { Shanghai }\end{array}$ & $\begin{array}{l}\text { Upper } \\
\text { middle } \\
\text { income }\end{array}$ & 2,025 & $7-13$ & $\begin{array}{c}\text { OWOB } \\
(\mathrm{BMI} \geq 24 \\
\left.\mathrm{kg} / \mathrm{m}^{2}\right) \\
\text { Chinese } \\
\text { adult BMI } \\
\text { reference }\end{array}$ & $\begin{array}{c}\text { OWOB } \\
\text { Chinese } \\
\text { school-age } \\
\text { BMI reference }\end{array}$ & $\begin{array}{l}\text { FC: } 2.26(1.78-2.86) \\
\text { MC: } 2.17(1.87-3.92) \\
\text { PC: } 4.36(3.16-6.01)\end{array}$ \\
\hline
\end{tabular}

OR, odds ratio; $\mathrm{Cl}$, confidence interval; OWOB, overweight and obese; IOTF, International Obesity Task Force; F, father; $\mathrm{M}$, mother; C, child; BMI, body mass index; FB, father-boy; FC, father-child; FG, father-girl; MB, mother-boy; MC, mother-child; MG, mother-girl; PC, parent-child; OW, overweight; OB, obesity; CDC, Centers for Disease Control and Prevention; WHO, World Health Organization; SD, standard deviation.

a) Most studies used the World Health Organization (WHO) definition of overweight (BMl 25-29.9 kg/m²), obesity (BMl $\geq 30 \mathrm{~kg} / \mathrm{m}^{2}$ ), underweight (BMl<18.5 kg/ $\mathrm{m}^{2}$ ), and OWOB $\left(\mathrm{BM} \geq 25 \mathrm{~kg} / \mathrm{m}^{2}\right.$ ) for parents. Other definitions are presented in the table. ${ }^{\mathrm{b}}$ In children, most studies used the International Obesity Task Force definition or the WHO definition. All definitions are indicated in the table.

high-income countries. Most studies used the WHO definition for parental weight status. In pediatric cases, the IOTF definition or WHO definition was used for child weight status. PC pairs and weight status combinations depend on study purpose, but OW or OWOB was more often used as the weight status for parents and children than was $\mathrm{OB}$ (number of data points for parents, $\mathrm{OWOB}=32, \mathrm{OW}=32, \mathrm{BMI}=21$, and $\mathrm{OB}=17$; for children, $\mathrm{OW}=45, \mathrm{OB}=40$, and $\mathrm{OWOB}=17$ ). The $\mathrm{OR}$ of reported $\mathrm{PC} \mathrm{OB}$ associations ranged from 0.98 to 24.3.

Table 2 shows PC OB associations. Most of the 23 studies showed a positive association in OB (87.3\%). Of all the PC pairs, $10 \mathrm{FC}$ pairs $(26.3 \%)$ and $3 \mathrm{MC}$ pairs $(6.5 \%)$ showed no significant association between parent and child $\mathrm{OB}$.

Most of the world regions showed a positive association between parent and child $\mathrm{OB}$, but there was no significant association between parent and child $\mathrm{OB}$ in 3 data points from North America (42.9\%) and in 3 data points from Africa (60\%). According to country income level, 4 data points from highincome countries $(7.7 \%)$ and 9 data points from middle- or low-income countries (17.6\%) showed no significant association between parent and child OB.

\section{Meta-analysis of parent and child association in $\mathrm{OB}$}

A meta-analysis based on data points from 23 studies found that parent and child OW or obese statuses were significantly associated (pooled OR, 1.97; 95\% CI, 1.85-2.10) (Table 2). However, there was considerable heterogeneity $(\mathrm{Q}=2295.87$, $d f=101, P<0.001)$.

Table 3 shows a meta-regression model predicting the parent and child $\mathrm{OB}$ association by child age, PC pair, parent and child weight status, world region, and country income level. The final meta-regression model was as follows:

$\log (\mathrm{OR}$ of parent and child association in $\mathrm{OB})=1.75+$ $0.06 \times$ child age $-0.92 \times \mathrm{FC}$ pair $-0.92 \times \mathrm{MC}$ pair $+0.55 \times$ parental $\mathrm{OB}+0.35 \times$ parental $\mathrm{OWOB}+0.22 \times$ child $\mathrm{OB}-$ $0.06 \times$ child OWOB $-0.83 \times$ Europe $-0.73 \times$ North America $-0.38 \times$ Latin America $-1.45 \times$ Middle East $-0.39 \times$ Africa + $0.43 \times$ country income level.

This study showed that type of PC pair, parent and child weight statuses, world region, and country's income level were 
Table 2. Association between and pooled estimates of parental and child obesity by parent-child pair type, weight status, world region, and country income level

\begin{tabular}{|c|c|c|c|c|c|}
\hline Variable & Total data & Not significant association & Positive association & Pooled OR (95\% Cl) & $1^{2}$ \\
\hline Total data & 102 & $13(12.7)$ & 89 (87.3) & 1.97 (1.85-2.10) & $95.6 \%$ \\
\hline Parent pair & 102 & & & & \\
\hline Parents & 18 & $0(0)$ & 18 (100) & $3.53(2.68-4.65)$ & $97.4 \%$ \\
\hline Father & 38 & $10(26.3)$ & $28(73.7)$ & $1.58(1.46-1.71)$ & $89.1 \%$ \\
\hline Mother & 46 & $3(6.5)$ & $43(93.5)$ & $1.65(1.54-1.76)$ & $90.7 \%$ \\
\hline Parental weight status & 81 & & & & \\
\hline OW & 32 & $6(18.8)$ & $26(81.3)$ & $2.18(1.90-2.49)$ & $85.9 \%$ \\
\hline OWOB & 32 & $3(9.4)$ & $29(90.6)$ & $2.29(2.02-2.60)$ & $56.8 \%$ \\
\hline $\mathrm{OB}$ & 17 & $1(5.9)$ & $16(94.1)$ & $4.07(2.89-5.74)$ & $95.7 \%$ \\
\hline Child weight status & 102 & & & & \\
\hline OW & 45 & $8(17.8)$ & $37(82.2)$ & $2.84(2.34-3.45)$ & $86.8 \%$ \\
\hline OWOB & 17 & $3(17.6)$ & $14(82.4)$ & $1.44(1.36-1.52)$ & $94.5 \%$ \\
\hline $\mathrm{OB}$ & 40 & $2(5)$ & $38(95)$ & $1.64(1.45-1.84)$ & $96.5 \%$ \\
\hline World regions & 102 & & & & \\
\hline Asia and pacific & 36 & $2(5.6)$ & $34(94.4)$ & $2.24(1.96-2.56)$ & $93.2 \%$ \\
\hline Europe & 33 & $3(9.1)$ & $30(90.9)$ & $2.11(1.91-2.32)$ & $96.9 \%$ \\
\hline Middle East & 10 & $2(20)$ & $8(80)$ & $1.63(1.28-2.08)$ & $97.7 \%$ \\
\hline North America & 7 & 3 (42.9) & $4(57.1)$ & $2.13(1.60-2.83)$ & $40.8 \%$ \\
\hline Latin America & 11 & $0(0)$ & $11(100)$ & $1.60(1.33-1.94)$ & $89.7 \%$ \\
\hline Africa & 5 & $3(60)$ & $2(40)$ & 1.60 (0.99-2.58) & $72.8 \%$ \\
\hline Country income level & 102 & & & & \\
\hline High & 52 & $4(7.7)$ & $48(92.3)$ & $2.29(2.10-2.51)$ & $95.9 \%$ \\
\hline Middle & 49 & 8 (16.3) & $41(83.7)$ & $1.73(1.58-1.90)^{a)}$ & $95.4 \%$ \\
\hline Low & 2 & $1(50)$ & $1(50)$ & & \\
\hline
\end{tabular}

OR, odds ratio; $\mathrm{Cl}$, confidence interval; OW, overweight; OWOB, overweight and obese; OB, obesity.

a) Middle- and low-income country pooled OR (95\% Cl).

important predictors of the PC OB associations.

This study found that the association between the child's weight status and that of both parents was stronger than that of either the father or mother alone (father $\beta \pm$ standard error $[\mathrm{SE}]$ : $-0.94 \pm 0.14, P<0.001$; mother $\beta \pm$ SE: $-0.93 \pm 0.14, P<0.001$ ).

This study also showed that OW status was associated more weakly with $\mathrm{PC} \mathrm{OB}$ than with $\mathrm{OB}$ or OWOB (OB: $\beta \pm \mathrm{SE}$ : $0.55 \pm 0.12, P<0.001$; OWOB $\beta \pm$ SE: $0.29 \pm 0.12, P=0.022$ ).

According to world region, Asia had a higher PC OB relation than did Europe or the Middle East (Europe $\beta \pm \mathrm{SE}$ : $-0.26 \pm 0.11, P=0.026$; Middle East $\beta \pm$ SE: $-0.95 \pm 0.17, P<$ 0.001). Furthermore, this study showed that high-income countries $(\beta \pm$ SE: $0.36 \pm 0.12, P=0.004)$ had a stronger PC OB relationship than middle- or low-income countries.

Pooled OR varied according to PC pair by country income, weight status, and world region as follows: (1) PC pairs by country income-pooled OR: 8.74 (95\% CI, 5.54-13.78) at PC pairs in high-income regions, 2.08 (95\% CI, 1.67-2.59) at PC pairs in middle- and low-income regions, 1.44 (95\% CI, 1.31$1.58)$ at FC pairs in high-income regions, 1.69 (95\% CI, 1.441.99) at FC pairs in middle- and low-income regions, 1.19 (95\% $\mathrm{CI}, 1.72-2.11)$ at $\mathrm{MC}$ pairs in high-income regions, and 1.47 (95\% CI, 1.34-1.61) at MC pairs in middle- and low-income regions (Figs. 3-5), (2) parental weight status-pooled OR: 4.07 (95\% CI, 2.89-5.74) in OB, 2.18 (95\% CI, 1.90-2.49) in OW, and 2.29 (95\% CI, 2.02-2.60) in OWOB; (3) child's weight status-pooled OR: 1.64 (95\% CI, 1.45-1.84) in OB, 2.84 (95\% CI, 2.34-3.45) in OW, and 1.44 (95\% CI, 1.36-1.52) in OWOB; (4) world region-pooled OR: 2.24 (95\% CI, 1.96-2.56) in Asia and Pacific, 2.11 (95\% CI, 1.91-2.32) in Europe, 2.13 (95\% CI, 1.60-2.83) in North America, 1.60 (95\% CI, 1.33-1.94) in Latin America, 1.63 (95\% CI, 1.28-2.08) in the Middle East, and 1.60 (95\% CI, 0.99-2.58) in Africa; (5) country income level-pooled OR: 2.29 (95\% CI, 2.10-2.51) in high-income and 1.73 (95\% CI, 1.58-1.90) in middle- and low-income countries. The results are shown in Table 2.

The Asia and Pacific region had stronger PC OB relationships compared to other regions. According to country income level, high-income countries were much more likely to report higher parent and child OB relationships than were middle- or lowincome countries.

\section{Publication bias}

A funnel plot was used to assess publication bias in the studies evaluating relation between parent and child OB. The funnel plot showed asymmetry, and Egger's test showed significant publication bias (intercept $=-0.23 ; 95 \%$ CI, -0.34 to $-0.12, z$ $=-3.94, P<0.0001$ ) (Supplementary materials). Therefore, we cannot exclude potential publication bias. Trim and fill methods are used to correct for publication bias in meta-analysis. ${ }^{37)}$ Using 
Table 3. Results of meta-regression: factors related with the parent-child association in obesity based on reported odds ratio of 23 studies

\begin{tabular}{|c|c|c|c|}
\hline Variable & $\beta$ & SE & $P$ value \\
\hline \multicolumn{4}{|l|}{ Child age } \\
\hline$<5 y r$ & 0.07 & 0.17 & 0.689 \\
\hline Included school-age & Reference & & \\
\hline \multicolumn{4}{|c|}{ Type of parent-child pairs } \\
\hline Parents & Reference & & \\
\hline Father & -0.94 & 0.14 & $<0.001$ \\
\hline Mother & -0.93 & 0.14 & $<0.001$ \\
\hline \multicolumn{4}{|c|}{ Parental weight status studied } \\
\hline Overweight & Reference & & \\
\hline Obesity & 0.55 & 0.10 & $<0.001$ \\
\hline OWOB & 0.29 & 0.12 & 0.022 \\
\hline \multicolumn{4}{|c|}{ Child weight status studied } \\
\hline Overweight & Reference & & \\
\hline Obesity & 0.18 & 0.10 & 0.066 \\
\hline OWOB & 0.29 & 0.12 & 0.435 \\
\hline \multicolumn{4}{|l|}{ Country region } \\
\hline Asia \& Pacific & Reference & & \\
\hline Europe & -0.26 & 0.11 & 0.026 \\
\hline North America & -0.16 & 0.19 & 0.386 \\
\hline Latin America & 0.13 & 0.15 & 0.400 \\
\hline Middle East & -0.95 & 0.17 & $<0.001$ \\
\hline Africa & -0.11 & 0.22 & 0.641 \\
\hline \multicolumn{4}{|c|}{ Country economic development } \\
\hline High & 0.36 & 0.12 & 0.004 \\
\hline Middle \& low & Reference & & \\
\hline
\end{tabular}

SE, standard error; OWOB, overweight and obese.

The outcome variable used in the meta-regression model was the odds ratio. A Stata Metareg command was used with the dummy variables of type of parent-child pair, parental and child weight status, country region, and categorical variables of child age and country income level.

Boldface indicates a statistically significant difference with $P<0.05$.

a trim and fill method, 47 prediction studies with results lower than the mean were excluded from analyses. Had these missing predictive studies been included in the meta-analysis, the pooled OR would have decreased to 1.30 (95\% CI, 1.13-1.51). But, the corrected effect estimates (pooled OR, 1.30; 95\% CI, 1.131.51) did not differ in direction, size, or statistical significance of the effect estimates before correction (pooled OR, 1.97; 95\% CI, 1.85-2.10).

\section{Discussion}

The risk of $\mathrm{OB}$ can pass from one generation to the next as a result of biological and/or environmental factors. Parents and children share about $50 \%$ of their genes, and these genetic factors likely explain some of the observed intergenerational association. In addition, parents and children share a common environment that can correlate with BMI and $\mathrm{OB}$ status, such as eating the same meals and engaging in similar physical activities. ${ }^{6}$ This meta-analysis demonstrated a significant $\mathrm{PC} \mathrm{OB}$
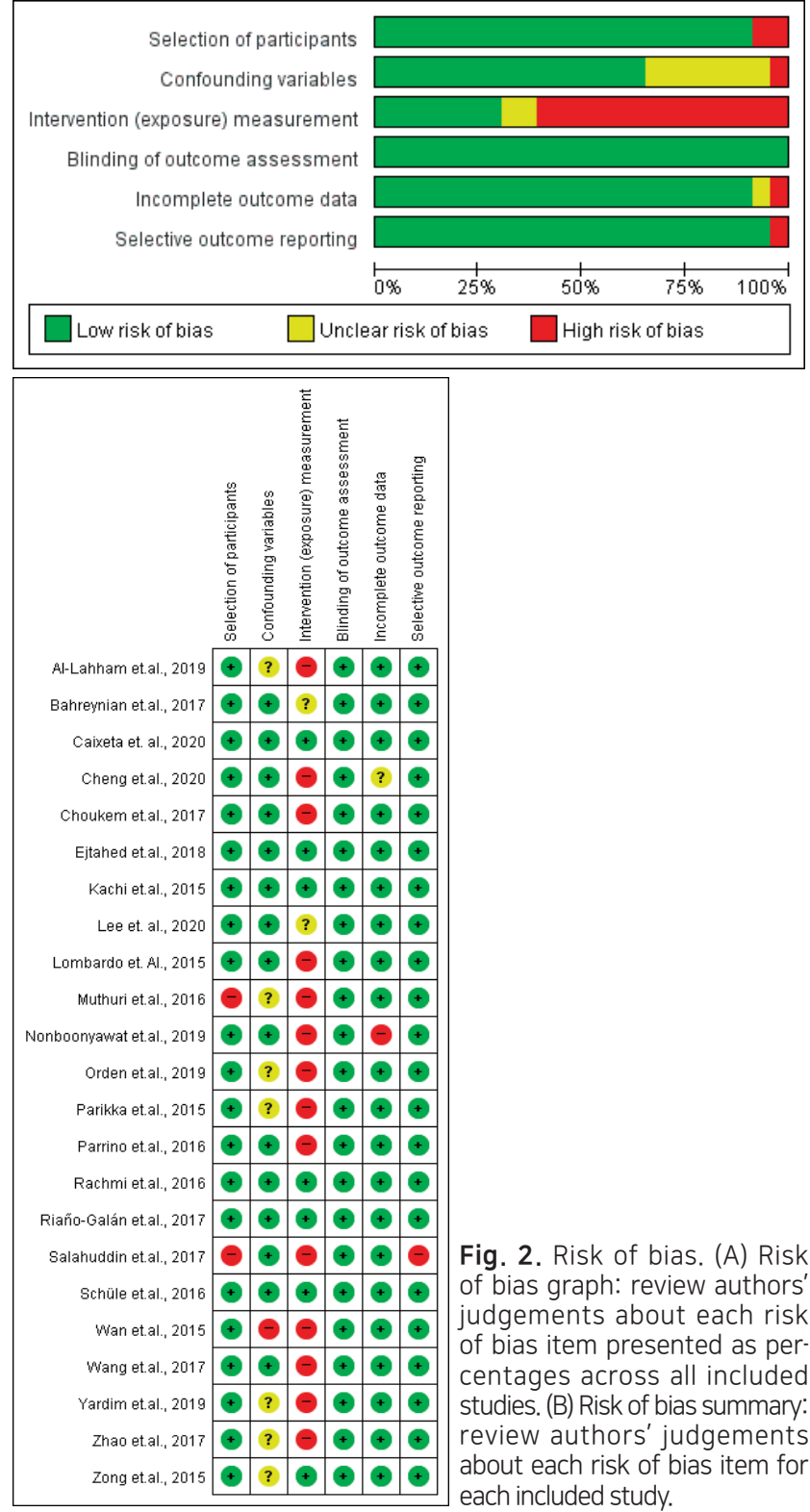

relationship (pooled OR, 1.97; 95\% CI, 1.85-2.10). Children with OW or obese parents were 1.97 times more likely to be OW or obese than children with healthy-weight parents. Studies performed in many countries have demonstrated many possible reasons for the body weight relationship between parents and their children. Epigenetic mechanisms such as DNA methylation demonstrate how inherited OB-related genes interact with environmental factors, which can lead to development of childhood OB. ${ }^{38)}$ Epigenetic changes can affect OB-related hormones such as such as leptin, insulin, and ghrelin and are dynamically regulated by nutritional and metabolic status. ${ }^{39)}$ Leptin plays a role in reducing food intake and decreasing body weight, ${ }^{40)}$ whereas decreased insulin receptors cause increases in food intake, adiposity, and peripheral insulin resistance, ${ }^{41)}$ and ghrelin promotes appetite, suppresses energy expenditure, and causes weight gain. ${ }^{42)}$ Furthermore, family lifestyles are an important risk factor for child $\mathrm{OB}$, and dietary behavior of the parents is 


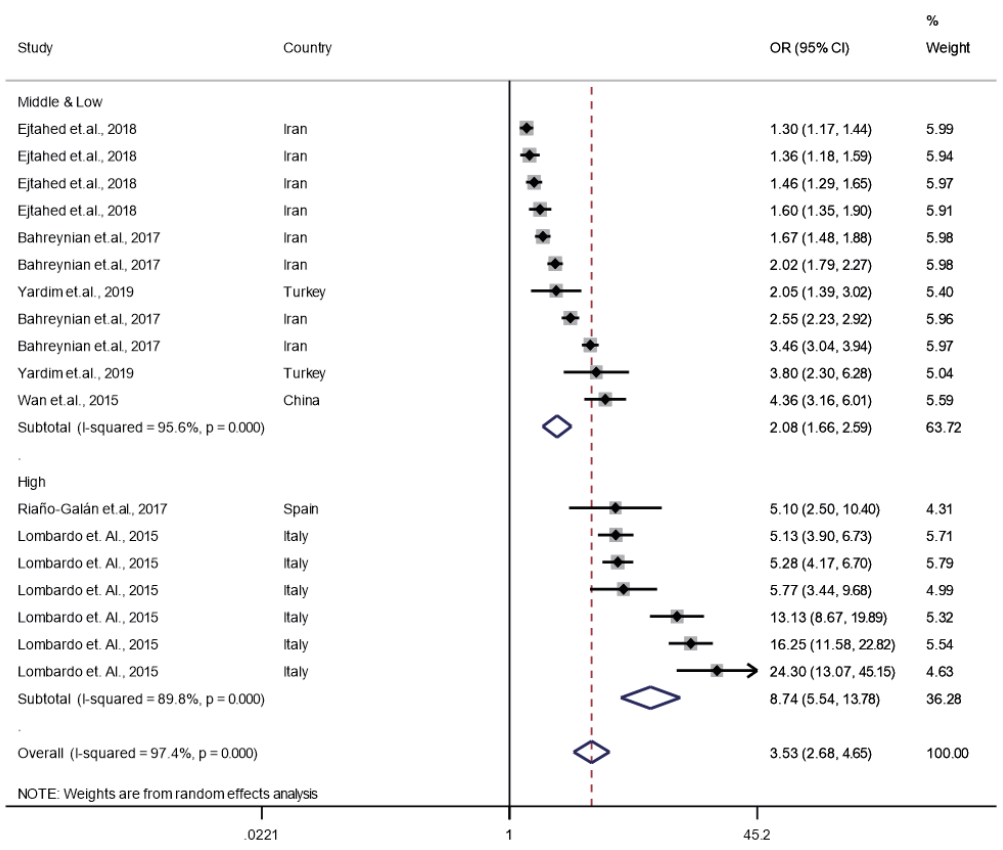

Fig. 3. Meta-analysis results of reported odds ratio (OR) of the correlation between parent and child obesity in 23 studies for parent-child pairs. Pooled estimation with 18 data points from 23 studies. Multiple OR for same studies reflect OR for different subgroups (middle- and low-income country OR: 2.08 [95\% Cl, 1.66-2.59]; test for heterogeneity: Cochrane $\mathrm{Q}=229.44$ [ $d f=10], P<0.001$; vs. high-income country OR: 8.74 [95\% Cl, 5.54-13.78]; test for heterogeneity: Cochrane $\mathrm{Q}=58.74[d f=6], P<0.001) . \mathrm{Cl}$, confidence interval; $d f$, degrees of freedom.

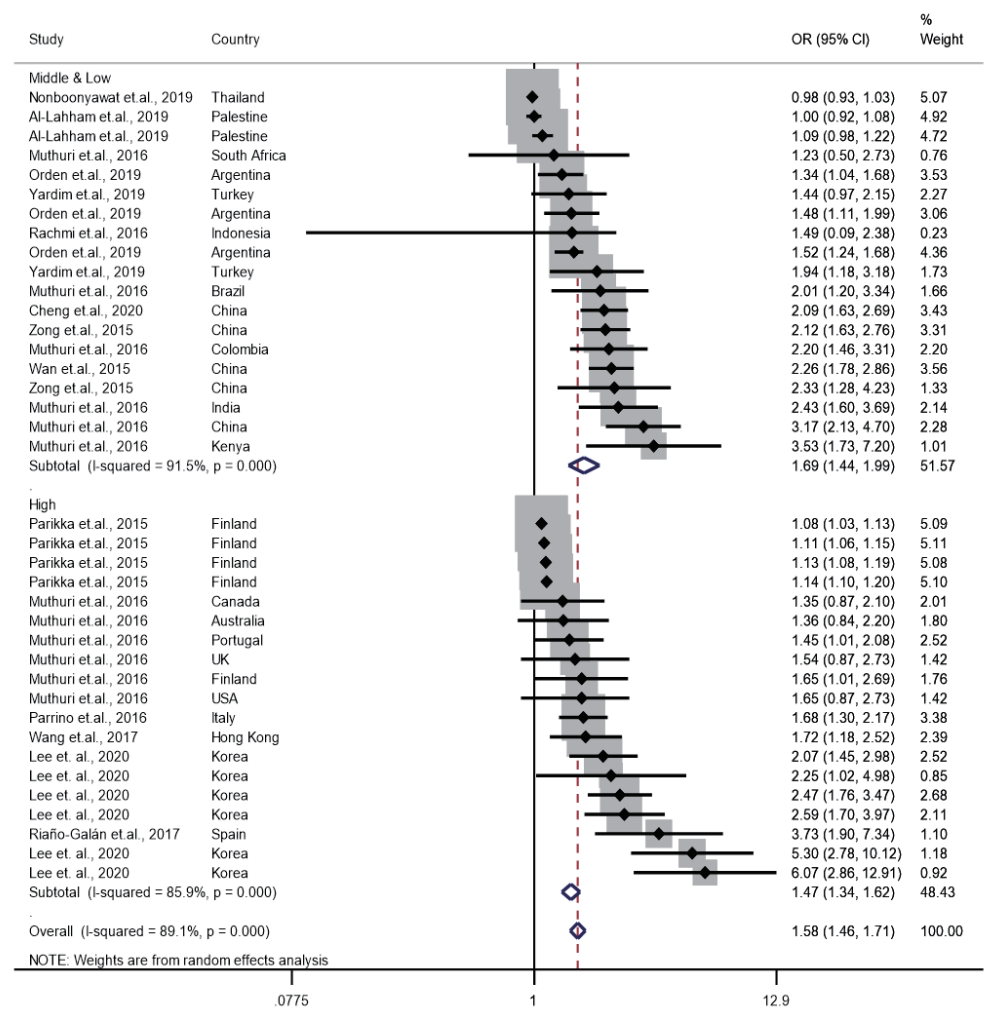

Fig. 4. Meta-analysis results of reported odds ratio (OR) of the correlation between parent and child obesity in 23 studies for the father-child pair. Pooled estimation with 38 data points from 23 studies. Multiple OR for same studies reflect OR for different subgroups (middle- and low-income country OR: 1.69 [95\% Cl, 1.44-1.99]; test for heterogeneity: Cochrane $\mathrm{Q}=212.63[d f=18], P<0.001$ vs. high-income country OR: 1.47 [95\% Cl, 1.34-1.62]; test for heterogeneity: Cochrane $Q=128.10[d f=18], P<0.001) . C l$, confidence interval; $d f$, degrees of freedom. 


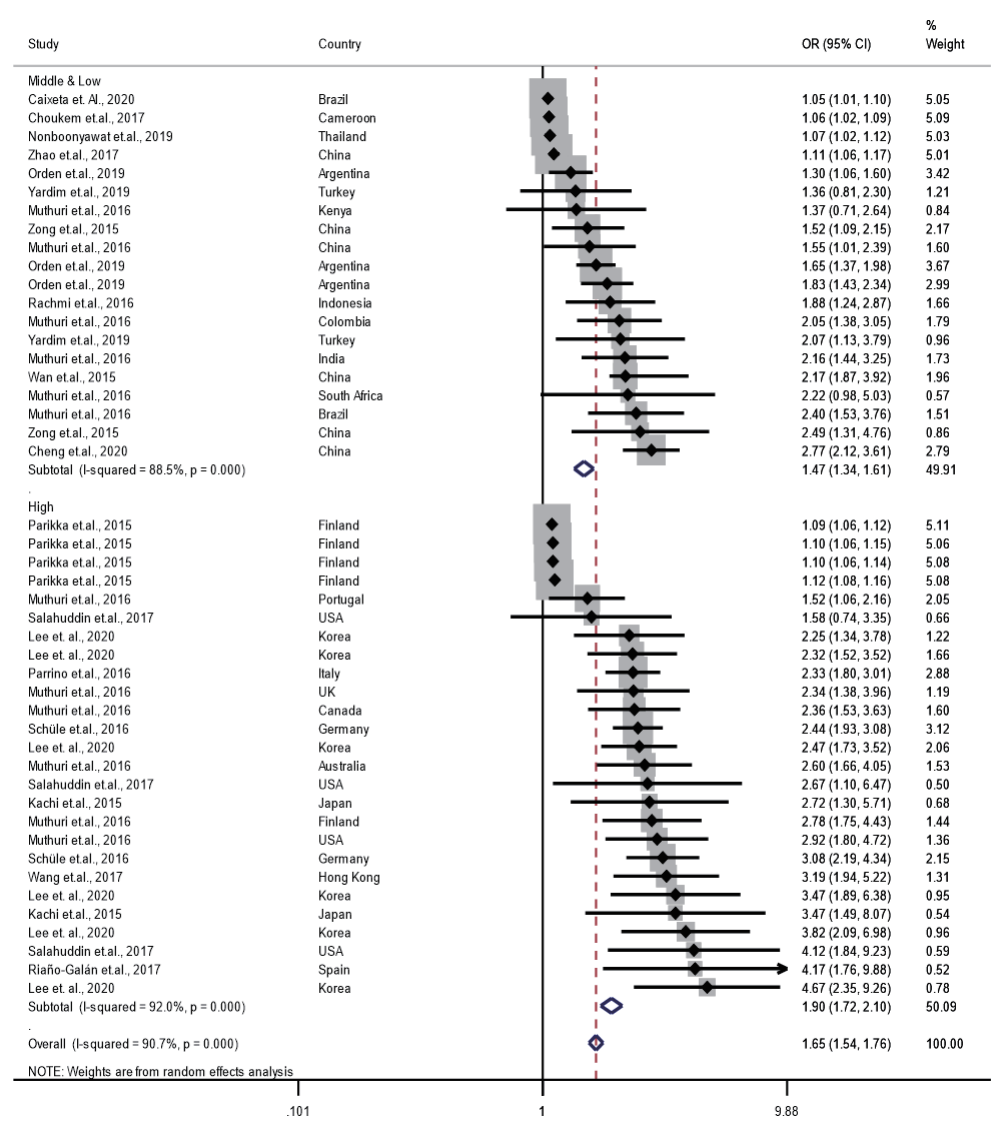

Fig. 5. Meta-analysis results of reported odds ratio (OR) of the correlation between parent and child obesity in 23 studies for the mother-child pair. Pooled estimation with 46 data points from 23 studies. Multiple OR for same studies reflect OR for different subgroups (middle- and low-income country OR: 1.47 [95\% Cl, 1.34-1.61]; test for heterogeneity: Cochrane $Q=165.63[d f=19], P<0.001$ vs. high-income country OR: 1.90 [95\% Cl, 1.72-2.10]; test for heterogeneity: Cochrane $Q=313.66[d f=25], P<0.001) . C l$, confidence interval; $d f$, degrees of freedom.

likely to determine a child's dietary patterns. ${ }^{43)}$

Meta-regression analysis showed that $\mathrm{PC} \mathrm{OB}$ association varied by parent and child pair (PC, FC, or MC), country income level, parent and child weight statuses (obese, OW, OWOB), and world region. The study showed that the OB relationship is stronger between PC pairs than between FC or MC pairs, meaning that children are more likely to be obese if both parents are OW or obese compared to children with only one parent (mother or father) who is OW or obese. These findings are consistent with a previous meta-analysis study and might be explained by a double genetic burden from having 2 OW or obese parents or as a result of a common family environment that is more likely to promote unhealthy weight gain in the family. ${ }^{44)}$ This study did not reveal any significant differences in OB between FC and MC pairs, but previous studies showed that maternal OW and $\mathrm{OB}$ status influenced childhood $\mathrm{OB}$ more than paternal OW and OB status. ${ }^{45,46)}$ Previous studies have shown that maternal $\mathrm{OB}$ is a more potent determinant of postpartum infant $\mathrm{BMI}$ growth and an indicator of $\mathrm{OB}$ risk, whereas paternal effects may appear later, possibly between 3 and 4 years of age. ${ }^{45)}$ In early childhood, eating habits may be predominantly shaped by the mother, which could be a factor in the differences previously shown between maternal and paternal OB. This meta-analysis did not include infants or children under 2 years of age, however, which may explain why no differences were observed between maternal and paternal OB.

According to country income level, our study showed that high-income countries were much more likely to report strong parent and child $\mathrm{OB}$ relationships than were middle- or lowincome countries. These "OB-causing" changes (such as economic growth, industrialization, mechanized transport, urbanization, and increased availability of abundant, inexpensive, and often undernourished foods) have been taking place in highincome countries since the beginning of the 20th century and are accelerating currently in low- and middle-income countries. ${ }^{47)}$ As multiple generations are exposed to high-risk environments, it is possible that intergenerational similarities in dietary habits and activity patterns are higher in high-income countries, while rapid generational transitions in diet and activity patterns persist in middle-income countries. ${ }^{44)}$ In high-income countries, the risk of childhood $\mathrm{OB}$ is greatest among lower socioeconomic groups. ${ }^{48)}$ Considering not only country income level but also family income, the United States (US) and Europe (high-income countries) could directly link wealth to OB by the middle of the 
20th century. ${ }^{47)}$ It was previously found that the richer was an individual, the more likely they were to be OW. Perhaps due to the abundance of inexpensive and highly available food as well as social changes, this association has been reversed in the present. Wealth in the US now tends to be inversely correlated with OB, and those are at or below the poverty line have the highest rates of $\mathrm{OB}^{49)}$

This study showed that Asia had higher PC OB relationships compared to Europe or the Middle East. A previous study showed that the overall prevalence of OW, including obese, school children in European countries was estimated at 20.5\%, where $15.6 \%$ were OW and $4.9 \%$ were obese. ${ }^{50)}$ The prevalence of OW and/or obese status was 24.5\% in Eastern Asia countries and $11.9 \%$ in the Western Asia regions, where the overall prevalence of $\mathrm{OB}$ and $\mathrm{OW}$ status were $5.8 \%$ and $11.2 \%$ in Asian children and $8.6 \%$ and $14.6 \%$ in adolescents, respectively. ${ }^{51)}$ Unlike the western societies that are promoting "individualism," the Asian society is "collectivistic" in that it promotes interdependence and co-operation, with families at the center of this social structure. ${ }^{52}$ Thus, Asian families tend to be more involved in care of one another and more involved in each other's lives. In Asian regions, the higher association between parental and child $\mathrm{OB}$ can be explained by the high $\mathrm{OB}$ rate in Asia coupled with the collectivistic family culture.

There are some limitations to this study. First, different cutoff points (IOTF, Centers for Disease Control and Prevention, $\mathrm{WHO}$, and country reference) were used to compared $\mathrm{OB}$ status in this meta-analysis, so it is difficult to directly compare across studies. Second, this study did not investigate the effects of family socioeconomic status, because published studies either did not include socioeconomic status factors or analyzed different socioeconomic status variables. Third, most of the included studies (21 of 23) were cross-sectional. Forth, the funnel plot presented an asymmetry, so publication bias cannot be fully excluded. In this study, asymmetry funnel plot with the scatter diagram is asymmetrical at the left lower bottom. This asymmetry indicates that smaller studies with negative findings are not published or not identified by the authors.

Further study should use longitudinal data to examine the parent and child relationships in $\mathrm{OB}$ and the related risk factors, including those related to family socioeconomic status such as family income, parental education level, and residential area.

The risk of childhood OB is greatly influenced by parental weight status. Most of the included studies reported a positive association between parent and child $\mathrm{OB}$, and this relationship varied depended on type of PC pair, parent and child weight statuses, world region, and national income level. Children with OW or obese parents are more likely to be OW or obese than those with parents of normal weight. Pediatric OB is a complex disorder due to environmental, biopsychosocial, genetic, and epigenetic factors. Further study is needed to uncover the biological and social factors of the PC OB relationship.

\section{Footnotes}

Supplementary material: Supplementary material can be found via https://doi.org/10.3345/cep.2020.01620

Conflicts of interest: No potential conflict of interest relevant to this article was reported.

ORCID:

Ju Suk Lee $\odot$ https://orcid.org/0000-0002-5600-3441

Mi Hyeon Jin 1 https://orcid.org/0000-0002-6436-2196

Hae Jeong Lee $(1$ https://orcid.org/0000-0003-2974-8365

\section{References}

1. World Health Organization. World health statistics 2020: monitoring health for the SDGs, sustainable development goals. Geneva (Switzerland): World Health Organization; 2020.

2. World Health Organization. Overweight prevalence among children under 5 years of age (\%) (JME) [Internet]. Geneva (Switzerland): World Health Organization; 2021 [cited 2021 Jan 4]. Available from: https:// www.who.int/data/gho/data/indicators/indicator-details/GHO/gho-jmeoverweight-prevalence.

3. World Health Organization. Obesity and overweight [Internet]. Geneva (Switzerland): World Health Organization; 2020 [cited 2020 Aug 31]. Available from: https://www.who.int/news-room/fact-sheets/detail/obesityand-overweight.

4. Mead E, Brown T, Rees K, Azevedo LB, Whittaker V, Jones D, et al. Diet, physical activity and behavioural interventions for the treatment of overweight or obese children from the age of 6 to 11 years. Cochrane 2017;6:Cd012651.

5. Must A, Strauss RS. Risks and consequences of childhood and adolescent obesity. Int J Obes Relat Metab Disord 1999;23 Suppl 2:S2-11.

6. Classen TJ, Thompson O. Genes and the intergenerational transmission of BMI and obesity. Econ Hum Biol 2016;23:121-33.

7. Moher D, Liberati A, Tetzlaff J, Altman DG. Preferred reporting items for systematic reviews and meta-analyses: the PRISMA statement. PLoS Med 2009;6:e1000097.

8. Gahagan S. Overweight and obesity. Nelson textbook of pediatrics. 21th ed. Philadelphia (PA): Elsevier, 2020. p. 345-58.

9. Centers for Disease Control and Prevention. Defining childhood weight status [Internet]. Atlanta (GA): Centers for Disease Control and Prevention; [cited 2020 Sep 4]. Available from: https:/www.cdc.gov/ obesity/childhood/defining.html.

10. Cole TJ, Bellizzi MC, Flegal KM, Dietz WH. Establishing a standard definition for child overweight and obesity worldwide: international survey. BMJ 2000;320:1240-3.

11. World Health Organization. WHO Expert committee on Physical Status: the use of and interpretation of anthropometry, report of a WHO expert committee. Geneva (Switzerland): World Health Organization; 1995.

12. Kim SY, Park JE, Lee YJ, Seo HJ, Sheen SS, Hahn S, et al. Testing a tool for assessing the risk of bias for nonrandomized studies showed moderate reliability and promising validity. J Clin Epidemiol 2013;66:408-14.

13. World Bank Group. World Bank Country and Lending Groups [Internet]. Washington, DC: World Bank Group; 2021 [cited 2020 Aug 21]. Available from: https://datahelpdesk.worldbank.org/knowledgebase/ articles/906519-world-bank-country-and-lending-groups.

14. Cheng L, Li Q, Hebestreit A, Song Y, Wang D, Cheng Y, et al. The associations of specific school- and individual-level characteristics with obesity among primary school children in Beijing, China. Public Health Nutr 2020;23:1838-45.

15. Caixeta HCV, Amato AA. Factors associated with overweight and 
abdominal obesity in Brazilian school-aged children: a comprehensive approach. Arch Endocrinol Metab 2020;64:445-53.

16. Lee HJ, Kim SH, Jin MH, Lee JS. Variability in sociodemographic factors and obesity in Korean children: a cross-sectional analysis of Korea National Health and Nutrition Examination survey data (2007-2015). Ann Epidemiol 2020;43:51-7.

17. Nonboonyawat T, Pusanasuwannasri W, Chanrat N, Wongthanavimok N, Tubngern D, Panutrakul P, et al. Prevalence and associates of obesity and overweight among school-age children in a rural community of Thailand. Korean J Pediatr 2019;62:179-86.

18. Yardim MS, Özcebe LH, Araz OM, Uner S, LiS, Unlu HK, et al. Prevalence of childhood obesity and related parental factors across socioeconomic strata in Ankara, Turkey. East Mediterr Health J 2019;25:374-84.

19. Al-Lahham S, Jaradat N, Altamimi M, Anabtawi O, Irshid A, AlQub M, et al. Prevalence of underweight, overweight and obesity among Palestinian school-age children and the associated risk factors: a cross sectional study. BMC Pediatr 2019;19:483.

20. Orden AB, Lamarque MS, Chan D, Mayer MA. Short sleep and low milk intake are associated with obesity in a community of school aged children from Argentina. Am J Hum Biol 2019;31:e23224.

21. Ejtahed HS, Heshmat R, Motlagh ME, Hasani-Ranjbar S, Ziaodini H, Taheri M, et al. Association of parental obesity with cardiometabolic risk factors in their children: the CASPIAN-V study. PLoS One 2018; 13:e0193978.

22. Choukem SP, Kamdeu-Chedeu J, Leary SD, Mboue-Djieka Y, Nebongo DN, Akazong C, et al. Overweight and obesity in children aged 3-13 years in urban Cameroon: a cross-sectional study of prevalence and association with socioeconomic status. BMC Obes 2017;4:7.

23. Riaño-Galán I, Fernández-Somoano A, Rodríguez-Dehli C, Valvi D, Vrijheid M, Tardón A. Proatherogenic lipid profile in early childhood: association with weight status at 4 years and parental obesity. J Pediatr 2017;187:153-7.e2.

24. Zhao Y, Wang L, Xue H, Wang H, Wang Y. Fast food consumption and its associations with obesity and hypertension among children: results from the baseline data of the Childhood Obesity Study in China Mega-cities. BMC Public Health 2017;17:933.

25. Salahuddin M, Pérez A, Ranjit N, Kelder SH, Barlow SE, Pont SJ, et al. Predictors of severe obesity in low-income, predominantly Hispanic/ Latino children: the Texas Childhood Obesity Research Demonstration Study. Prev Chronic Dis 2017;14:E141.

26. Wang JJ, Gao Y, Lau PWC. Prevalence of overweight in Hong Kong Chinese children: its associations with family, early-life development and behaviors-related factors. J Exerc Sci Fit 2017;15:89-95.

27. Bahreynian M, Qorbani M, Khaniabadi BM, Motlagh ME, Safari O, Asayesh $\mathrm{H}$, et al. Association between obesity and parental weight status in children and adolescents. J Clin Res Pediatr Endocrinol 2017;9:111-7.

28. Schüle SA, von Kries R, Fromme H, Bolte G. Neighbourhood socioeconomic context, individual socioeconomic position, and overweight in young children: a multilevel study in a large German city. BMC Obes 2016;3:25.

29. Parrino C, Vinciguerra F, La Spina N, Romeo L, Tumminia A, Baratta R, et al. Influence of early-life and parental factors on childhood overweight and obesity. J Endocrinol Invest 2016;39:1315-21.

30. Rachmi CN, Agho KE, Li M, Baur LA. Stunting, underweight and overweight in children aged 2.0-4.9 years in Indonesia: prevalence trends and associated risk factors. PLoS One 2016;11:e0154756.

31. Muthuri SK, Onywera VO, Tremblay MS, Broyles ST, Chaput JP, Fogelholm M, et al. Relationships between parental education and overweight with childhood overweight and physical activity in 9-11 year old children: results from a 12-country study. PLoS One 2016;11: e0147746.

32. Lombardo FL, Spinelli A, Lazzeri G, Lamberti A, Mazzarella G, Nardone $\mathrm{P}$, et al. Severe obesity prevalence in 8 - to 9-year-old Italian children: a large population-based study. Eur J Clin Nutr 2015;69:603-8.

33. Parikka S, Mäki P, Levälahti E, Lehtinen-Jacks S, Martelin T, Laatikainen T. Associations between parental BMI, socioeconomic factors, family structure and overweight in Finnish children: a path model approach. BMC Public Health 2015;15:271.
34. Kachi Y, Otsuka T, Kawada T. Socioeconomic status and overweight: a population-based cross-sectional study of Japanese children and adolescents. J Epidemiol 2015;25:463-9.

35. Zong XN, Li H, Zhang YQ. Family-related risk factors of obesity among preschool children: results from a series of national epidemiological surveys in China. BMC Public Health 2015;15:927.

36. Wan Y, Xu R, Feng H, Zhou Y, Zhang X, Lu L, et al. Is parental body weight related with their children's overweight and obesity in Gao Hang Town, Shanghai? Asia Pac J Clin Nutr 2015;24:509-14.

37. Duval S, Tweedie R. Trim and fill: a simple funnel-plot-based method of testing and adjusting for publication bias in meta-analysis. Biometrics 2000;56:455-63.

38. Herrera BM, Keildson S, Lindgren CM. Genetics and epigenetics of obesity. Maturitas 2011;69:41-9.

39. Bouret S, Levin BE, Ozanne SE. Gene-environment interactions controlling energy and glucose homeostasis and the developmental origins of obesity. Physiol Rev 2015;95:47-82.

40. Halaas JL, Boozer C, Blair-West J, Fidahusein N, Denton DA, Friedman JM. Physiological response to long-term peripheral and central leptin infusion in lean and obese mice. Proc Natl Acad Sci US A 1997;94:887883.

41. Obici S, Feng Z, Karkanias G, Baskin DG, Rossetti L. Decreasing hypothalamic insulin receptors causes hyperphagia and insulin resistance in rats. Nat Neurosci 2002;5:566-72.

42. Nakazato M, Murakami N, Date Y, Kojima M, Matsuo H, Kangawa $\mathrm{K}$, et al. A role for ghrelin in the central regulation of feeding. Nature 2001;409:194-8.

43. Sung-Chan P, Sung YW, Zhao X, Brownson RC. Family-based models for childhood-obesity intervention: a systematic review of randomized controlled trials. Obes Rev 2013;14:265-78.

44. Wang Y, Min J, Khuri J, Li M. A systematic examination of the association between parental and child obesity across countries. Adv Nutr 2017; 8:436-48.

45. Linabery AM, Nahhas RW, Johnson W, Choh AC, Towne B, Odegaard $\mathrm{AO}$, et al. Stronger influence of maternal than paternal obesity on infant and early childhood body mass index: the Fels Longitudinal Study. Pediatr Obes 2013;8:159-69.

46. Lee HJ, Kim SH, Choi SH, Lee JS. The association between socioeconomic status and obesity in korean children: an analysis of the Fifth Korea National Health and Nutrition Examination Survey (2010-2012). Pediatr Gastroenterol Hepatol Nutr 2017;20:186-93.

47. Hruby A, Hu FB. The epidemiology of obesity: a big picture. Pharmacoeconomics 2015;33:673-89.

48. World Health Organization. Report of the Commission on Ending Childhood Obesity [Internet]. Geneva (Switzerland): World Health Organization; 2016 [cited 2020 Aug 19]. Available from: https://www. who.int/publications/i/item/9789241510066.

49. Levine JA. Poverty and obesity in the U.S. Diabetes 2011;60:2667-8.

50. Olaya B, Moneta MV, Pez O, Bitfoi A, Carta MG, Eke C, et al. Countrylevel and individual correlates of overweight and obesity among primary school children: a cross-sectional study in seven European countries. BMC Public Health 2015; 15:475.

51. Mazidi M, Banach M, Kengne AP, Lipid, Blood Pressure Meta-analysis Collaboration G. Prevalence of childhood and adolescent overweight and obesity in Asian countries: a systematic review and meta-analysis. Arch Med Sci 2018;14:1185-203.

52. Chadda RK, Deb KS. Indian family systems, collectivistic society and psychotherapy. Indian J Psychiatry 2013;55:S299-309.

How to cite this article: Lee JS, Jin MG, Lee HJ. Global relationship between parent and child obesity: a systematic review and meta-analysis. Clin Exp Pediatr 2022;65:35-46. https://doi.org/10.3345/cep.2020.01620 